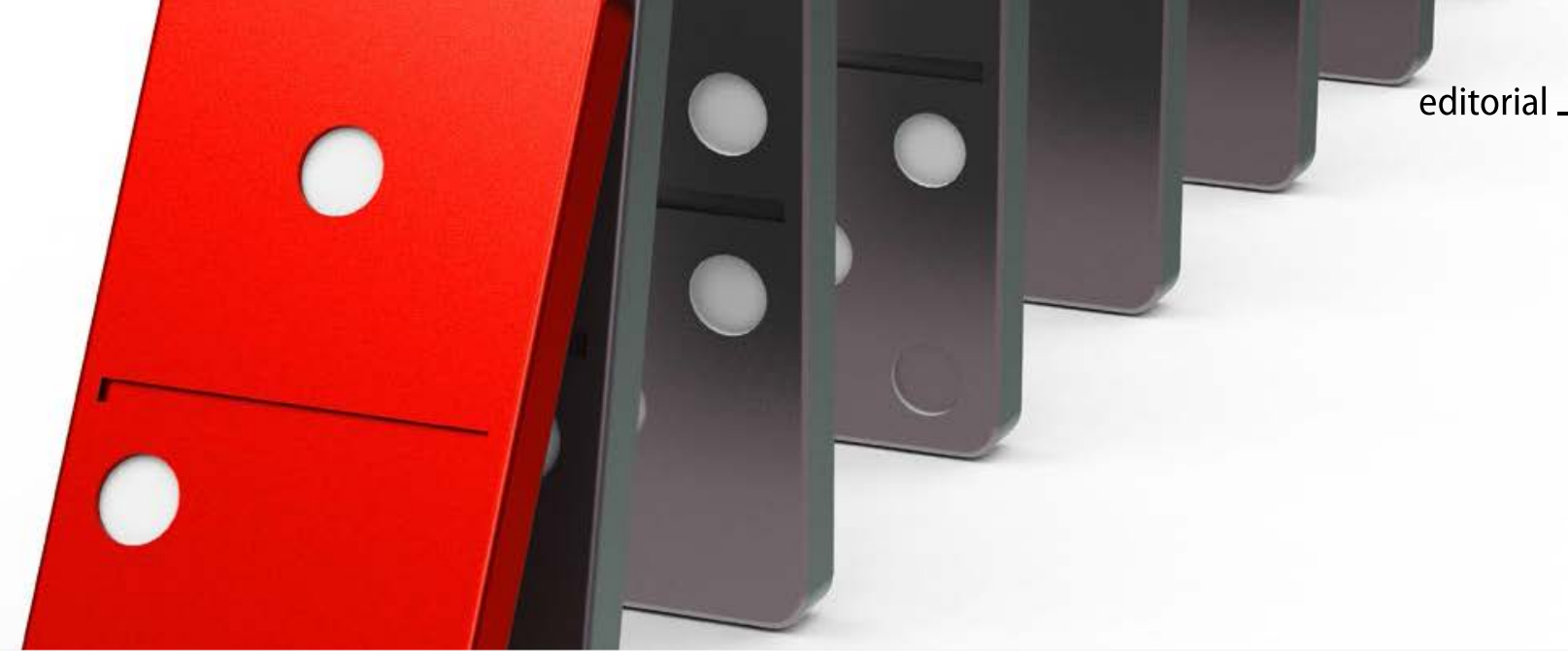

\title{
Punktwert sofort anpassen
}

Liebe Kolleginnen und Kollegen,

die Lüge hat vor drei Jahren begonnen: Am 1. Januar 2012 trat die GOZ-Novelle 2012 in Kraft. Eine Novelle, die schon damals weder den Namen verdient hatte noch weit genug ging. Wie schon in der Vergangenheit verweigerte die Bundesregierung auch vor drei Jahren den Zahnärzten die Punktwertanpassung an die Kostenentwicklung. Stattdessen wurde lediglich eine punktuelle Neustrukturierung und Neubewertung einzelner Leistungen vorgenommen. Das aber trägt nicht den gestiegenen Praxis-, Personal-, Material- und Hygienekosten Rechnung.

Der Punktwert hat die gesetzlich festgelegte Funktion, die wirtschaftliche Preisentwicklung widerzuspiegeln. Das legt $₫ 15$ des Zahnheilkundegesetzes fest: „Dabei ist den berechtigten Interessen der Zahnärzte [...] Rechnung zu tragen.“ Nun, da aber klar ist, dass die Bundesregierung keinen dringenden Handlungsbedarf für eine GOZ-Anpassung sieht, müssen wir davon ausgehen, dass es auf absehbare Zeit keine Anhebung des Punktwertes geben wird.

\section{Bezeichnung „angemessen“ in keiner Weise verdient}

Damit schneidet die Bundesregierung die Zahnärzte von der allgemeinen guten wirtschaftlichen Entwicklung in Deutschland ab. Ein Umstand übrigens, den es bei anderen freien Berufen so nicht gibt. Es ist zynisch, wenn die Bundesregierung jetzt in ihrer Stellungnahme feststellt, dass es anhand der dargestellten Auswirkungen der GOZ-Novelle 2012 derzeit keinen dringenden Handlungsbedarf für eine Änderung der GOZ gibt.

Kein Politiker, kein Angestellter, kein Rechtsanwalt, keine Mitarbeiterin in unserer Praxis würde es hinnehmen, 27 Jahre lang den gleichen Lohn oder das gleiche Gehalt zu bekommen. Es kann nicht sein, dass die GOZ zu einer Erstattungsordnung mutiert und sich an der finanziellen Situation der Kostenträger orientiert. Ein Punktwert der seit 1988 (!) bei 5,6241 Cent stagniert, hat die Bezeichnung ,angemessen“ in keiner Weise verdient. Von der seit 1988 aufgelaufenen Teuerungsrate von 75 Prozent ganz zu schweigen. Wenn die Politik weiterhin möchte, dass wir Zahnärzte qualitativ hochwertig arbeiten und alle Möglichkeiten der modernen Diagnose und Therapie nutzen - zum Wohle des Patienten - dann geht das auf Dauer nur mit einer angemessenen Honorierung. Deshalb fordert der FVDZ eine sofortige Punktwertanhebung auf elf Cent. Wir müssen zu einer Gebührenordnung statt einer Erstattungsordnung zurückkehren.

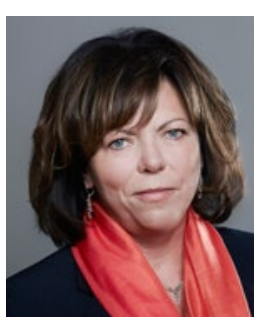

Kerstin Blaschke

Bundesvorsitzende des FVDZ

Darüber hinaus fordern wir, dass keine Leistungen unter BEMA-Niveau liegen. Gegenwärtig gibt es bereits mehr als 60 Leistungen, die bei einem Faktor 2,3 unter diesem Niveau liegen. Tendenz steigend! Eine GOZ-Novelle muss neue zeit- und materialintensivere Behandlungsmethoden in der Honorierung berücksichtigen, wie beispielsweise Zeit und Art der Ausführung bei unterschiedlichen Materialien (Kronenaustausch, Aufbaufüllungen). Die Vergütung bei zeitintensiven, alltäglichen Leistungen, wie zum Beispiel der Beratung und der vom Gesetzgeber geforderten Erstellung alternativer Behandlungspläne, muss deutlich angehoben werden.

Die Benachteiligung der Zahnärzte im Vergleich zu den Ärzten muss endlich abgeschafft werden. Das heißt: Bei gleicher Leistung (zum Beispiel digitales Röntgen) muss die gleiche Honorierung gelten.

\section{Nicht bis 2017 vertrösten lassen}

Wir können uns nicht bis 2017 vertrösten lassen, bis das Ergebnis der nächsten Evaluierung ins Haus steht. Wir müssen heute für die Honorarverbesserungen durch eine kontinuierliche Punktwert-Anpassung an einen geeigneten Preis-Index streiten. Deshalb fordert der Freie Verband eine nachhaltige Verbesserung der Honorarsituation für die Praxen. Für unsere Forderungen müssen wir die Reihen der Zahnärzte schließen und gemeinsam kämpfen - auch für die künftige Zahnärztegeneration.

Ihre Kerstin Blaschke

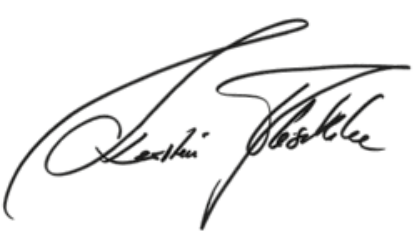

